\title{
Influential authorities for vaccination policies and barriers to implementing standing orders for influenza vaccination among nursing facilities in 14 states, 2000-2002
}

\author{
Barbara Bardenheier ${ }^{1}$, Abigail Shefer ${ }^{1}$, Stefan Gravenstein ${ }^{2}$, Carolyn Furlow ${ }^{1}$, \\ Carol J. Rowland Hogue ${ }^{3}$ \\ ${ }^{1}$ Centers for Disease Control and Prevention, National Center for Immunization and Respiratory Diseases, Atlanta, GA,USA \\ ${ }^{2}$ Quality Partners of Rhode Island and Brown University, Providence, RI, USA \\ ${ }^{3}$ Emory University, Atlanta, GA, USA
}

\section{Email address:}

bfb7@cdc.gov (B. Bardenheier), ams7@cdc.gov (A. Shefer), sgravensteinwork@gmail.com (S. Gravenstein), ijr1@cdc.gov (C. Furlow),CHOGUE@emory.edu (C. Hogue)

\section{To cite this article:}

Barbara Bardenheier, Abigail Shefer, Stefan Gravenstein, Carolyn Furlow, Carol J. Rowland Hogue. Influential Authorities for Vaccination Policies and Barriers to Implementing Standing Orders for Influenza Vaccination among Nursing Facilities in 14 States, $2000-2002$. American Journal of Theoretical and Applied Statistics. Vol. 1, No. 1, 2012, pp. 1-11. doi: 10.11648/j.ajtas.20120101.11

\begin{abstract}
To assess barriers to implementing standing order protocols (SOP) for vaccinations and influential authorities in making vaccination decisionswith the proportion of black residents, and vaccination coverage in nursing homes. The Centers for Medicare \& Medicaid Services and the Centers for Disease Control and Prevention in 2000-2002 surveyed approximately 280 nursing homes in14 states. Data from the On-line Survey and Certification Reporting System were included. A demonstration project to adopt SOPs for vaccination and to assess barriers. Factor analysis and structural equation models were used to assess relationships ofbarriers and influential authorities to implementing SOPs. External facility concerns are barriers to implementing SOPs $(p=.031)$, and nursing homes with higher proportions of black residents are more likely to report those concerns. The medical director and the facility administrator are the most influential authorities determining whether SOPs are implemented. The Quality Improvement Organization (QIO) and the state certification surveyor also played important roles in influencing staff making vaccination decisions. The state's QIO and the state certification surveyor may play important roles in addressingconcerns about staff's authority to vaccinate under SOPs.Barriers external to the nursing home may play a more important role than internal facility barriers.
\end{abstract}

Keywords: Vaccination, Nursing Home, Standing Orders

\section{Introduction}

Although annual influenza vaccination has been a longstanding national recommendation for nursing home residents, vaccination coverage has remained well below the Healthy People goal of 90\%.(1-4) One strategy known to increase coverage in facilities is standing order protocols (SOPs). SOPs for vaccination authorize nurses and other healthcare personnel, where allowed by state law, to assess patient'svaccination status and administer vaccinations according to a protocol approved by the institution, a physician, or other attending provider without the need for a physician's examination or direct order at the time of the interaction. $(5 ; 6 ; 6 ; 7)$ SOPs not only save time for physicians or other prescribers, but also reduce the number of missed opportunities for vaccination. Although the Advisory Committee on Immunization Practices (ACIP) and the Task Force on Community Preventive Services recommended SOPs for influenza vaccination, $(5 ; 6)$ only $40 \%$ of nursing home residents reportedly lived in homes with such protocols according to the 2004 National Nursing Home Survey (NNHS). (8).

In 2002 we surveyed nursing homes in 14 states regarding use of SOPs for influenza vaccination, and found that certain characteristics were associated with adoption of this evidence-based strategy (e.g., government-owned or nonprofit, not affiliated with a chain, and dually certified by 
the Centers for Medicare \& Medicaid Services (CMS) for both Medicare and Medicaid). (9) From the 2004 NNHS data, we also found proprietary (i.e., for profit) nursing homes and homes affiliated with a multi-facility chain both had a higher prevalence of black residents than white residents, and were less likely to have SOPs. (10) Another analysis of 2004 NNHS data found that use of standing order protocols (SOPs) was associated with increased influenza vaccination coverage and that the resident's race strongly confounded that relationship among nursing home residents. (8) That finding inspired a studyusing the 2004 NNHS data which reported a non-significant difference in influenza vaccination between white and black residents of homes with SOPs compared to a statistically significant difference between white and black residents of homes without SOPs $(p=.009)$. (10) .

Little evidence has been reported to establish which authorities most influence influenza vaccination policies in nursing homes and what are the main barriers to their implementation. To our knowledge, our previous analysis was the only study that found facility staff reported legal concerns such as liability for the facility and lacking legal authority as significant barriers to implementing SOPs. (9) Importantly, at the time of our study, CMS (Medicare) did not reimburse nursing homes with SOPs for the influenza vaccine yet lack of reimbursement was not found to be a significant barrier.

Because of the low prevalence in use of SOPs, last assessed at a national level in 2004, we sought to further examine influential authorities and barriers to their implementation. We used previously collected data from a demonstration project to improve uptake of SOPs among nursing homes (results of project's impact have been published elsewhere). (9;10) The objectives of the present study include: 1) whether observed variables interrelate to identify and confirm the following underlying factors: types of authorities most influential in vaccination policies, barriers to implementing SOPs, and residents' funding as a system of resources, 2) if these underlying factors are concomitantly associated with each other, the racial composition of the facility, and with implementing SOPs, and 3) if these underlying factors are directly or indirectly, via implementation of SOPs, associated with vaccination coverage.

\section{Methods}

From 2000-2002 the Centers for Disease Control and Prevention (CDC), CMS, and CMS' Quality Improvement Organizations (QIO) conducted a demonstration project called The Immunization Standing Orders Program Project. QIOs conduct quality improvement activities across healthcare settings and providers nationally, and the goal of this project was for the QIOs to promote SOPs for vaccination among nursing facilities in order to increase vaccination coverage among residents. Thirteen states (Florida, Hawaii, Idaho, Kentucky, Massachusetts, Minnesota, Montana, New Mexico, Ohio, Pennsylvania, Wisconsin, South
Carolina, and Nevada) and the District of Columbia participated. Twenty facilities were selected in each state. Before the start of the project, no states were known to have laws or regulations restricting the use of SOPs; however there was a federal law that prevented Medicare reimbursement for the cost of vaccination in facilities that used SOPs. Facilities were selected using a stratified random sampling design by facility size and the type of vaccination program. QIOs were responsible for onsite data collection and program promotion. Further information on this project has been published elsewhere. (11) Analysis of data from this project was approved by Emory University's Institutional Review Board.

\subsection{Demonstration Project Facility Survey Data}

Facilities were first surveyed November 1, 2000 through January 31, 2001 before the intervention, with a second survey administered during the same time period in 20012002. In this paper we used survey data from both years for the factor analysis but the final structural equation models used the second year data only. The survey administered during the second year of the demonstration project collected information on whether or not facilities had SOPs, as well as the importance of a variety of authoritative persons and organization on shaping the facility's immunization program and the barriers to implementing an SOP for vaccination.QIOs distributed the surveys to the facilities in their jurisdiction and collected the completed surveys; survey data were subsequently double-entered into the study database. The staff member at each facility who completed the survey varied: Director of Nursing (40.6\%), Infection Control Coordinator (29.9\%), Facility Administrator (10.3\%), Assistant Director of Nursing (9.0\%), and other facility staff $(10.2 \%)$.

Vaccination program activities were defined as "SOP" or "non-SOP" programs. For the purpose of our survey, "SOP" was defined as programs that authorize nurses, physician assistants, and pharmacists, where allowed by state law, to assess a client's vaccination status and administer vaccinations according to an institution- or physician-approved protocol without the need for a physician's examination or direct order at the time of the interaction. (5) The following vaccination programs were operationally defined as "nonSOP": "advanced orders" (i.e., an individual physician authorizes appropriate nursing staff to immunize his/her patients by his/her approved protocol without the need for an additional written or verbal order); preprinted admission orders (i.e. , standardized forms included in admission package for personal physician signature which may address future as well as current vaccination needs); reminders/education (procedures in place for educating and/or reminding physicians and residents on importance of vaccinations); and usual care (residents are immunized upon request and/or upon personal physician's individual discretion, and require physician's order for each vaccination).

\subsection{Other Facility Data}


The authority or authorities that influence vaccination policies were addressed through a single question, "How important are the following authorities in shaping the facility's immunization policies? Please check 1 = very important; 2 = important; $3=$ somewhat important; $4=$ not important; 5 = don't know." The following authorities were listed: ACIP, facility's medical director, quality improvement organization, state LTCF certification surveyor, state public health department, facility administrator, corporate leadership, director of nursing, and infection control coordinator. For ease of interpretation in the factor analysis, we rescaled these variables from $1=$ "don't know" to $5=$ "very important."

Barriers were assessed with a single question, "Rate the significance of barriers that prevent implementation of standing order programs for vaccinating LTCF residents.Please check for 1 = very significant; 2 = significant; 3 = somewhat significant; $4=$ not significant; $5=$ don't know." The following barriers were listed: staff lack the legal authority, lack of support of facility leadership, physicians need education about standing order programs, cost of the program (e.g., staffing requirements), low reimbursement, other priorities of staff time, medical liability concerns for the facility, and concern about inappropriate vaccination of the resident. For ease of interpretation in the factor analysis, we rescaled these variables from $1=$ "don't know" to 5 = "very significant".

The On-line Survey and Certification Reporting System (OSCAR) is an administrative database containing information on all Medicare or Medicaid licensed LTCFs in the U.S. The OSCAR variables used in this analysis included ownership (government, nonprofit, or proprietary), facility size (i.e., number of beds), whether the facility had any substandard assessments, (12) whether the facility was hospital-administered, type of facility (i.e., skilled nursing facility (a licensed physician supervises each patient's care and a nurse or other medical professional is almost always on the premises) or nursing facility- Medicare and/or Medicaid certified), and if the facility was independent or part of a multi-facility chain.

Vaccination coverage for the nursing home was based on a sample of residents. Residents eligible for inclusion lived in the facilities for any period of time during the influenza season from November 1, 2001 through January 31, 2002. One hundred residents (or all residents in facilities with < 100 residents) were randomly chosen from each facility and vaccination histories were collected through on-site and archived chart abstractions. Vaccination status was aggregated for each nursing home. Racial composition of the LTCF was determined from CMS's Minimum Data Set (MDS). MDS is part of the resident assessment instrument (RAI) completed by nurses and used for assessment and care screening. (13) Instructions for completing residents' assessments include reporting race "within which the resident places self." (12) .

\subsection{Statistical Models}

We used structural equation modeling with latent variables (i.e., underlying factors) to evaluate the effects on vaccination coverage in nursing homes of: barriers to implementing SOPs; and facility staff influential in decisions about use of standing orders for vaccinations. In general, structural equation models reduce measurement error through multiple indicators per latent variable, facilitate testing models with multiple dependent variables, and allow tests about overall models rather than individual factors. In addition, structural equation models examine factors' direct and indirect effects on dependent variables while allowing examination of complex associations among multiple factors. (13) Hence, the independent factors and combined mediated relationships can be discovered.

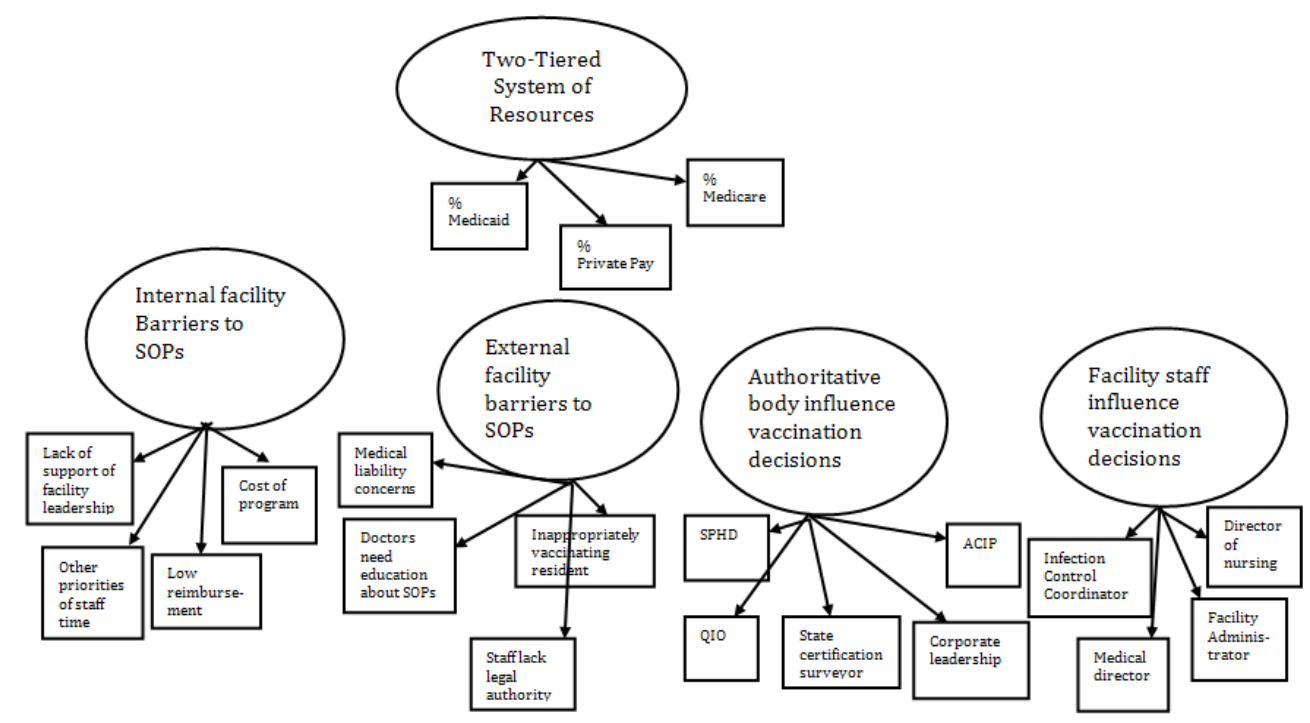

Figure 1. Conceptual Model of Barriers to implementing standing orders for vaccinations, influential authorities for vaccination policies and two-tiered system of resources. 
We conducted an exploratory factor analysis to tentatively identify underlying factors that define barriers to implementing standing vaccination orders and influential authorities for vaccination policy decisions. Then we used confirmatory factor analysis (CFA) to determine if the indicator variables that made up the latent variables were consistent between survey years. [Figure 1] CFA was assessed using the model fit criteria:(13) Comparative Fit Index $(\mathrm{CFI})>0.95$; root Mean Square Error of Approximation (RMSEA) < 0.08; and the Standardized Root Mean Square Residual (SRMR) $<0.06$.
We examined the results of the factor analysis with additional covariates by fitting a structural equation model. The latent variables confirmed in the factor analysis define the measurement portion of the structural equation model; the relationships among the latent variables and the additional covariates or other observed variables define the model's structural portion. Additional covariates included: proportion of black residents in the facility; implementation of SOPs; and vaccination coverage.The study's conceptual model appears in Figure 2.

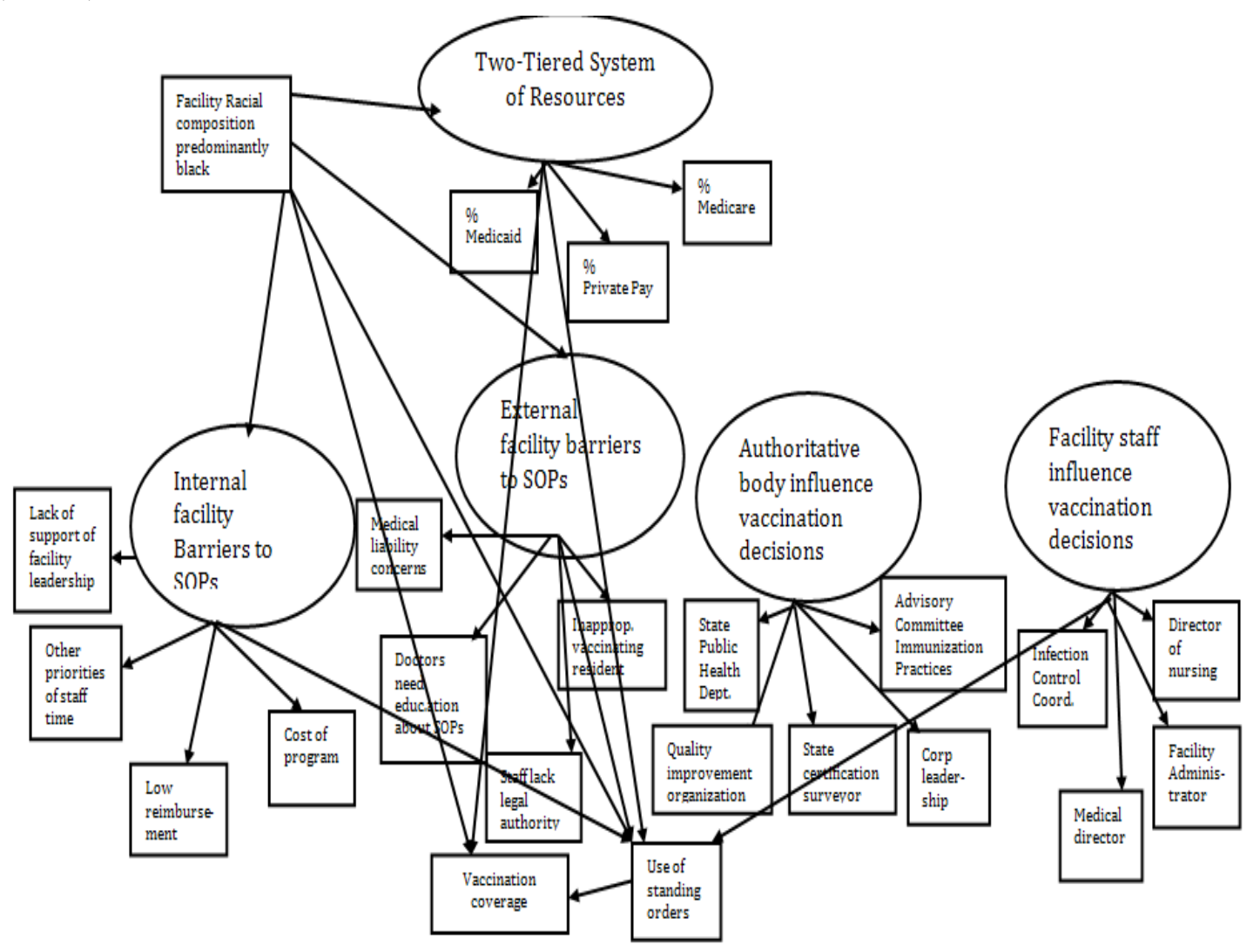

Figure 2. Conceptual Model of Relationships tested in Structural Equation Model.

A priori, we predicted eleven paths to directly/indirectly affect implementation of standing orders and/or vaccination coverage. Nursing homes with a larger proportion of black residents were anticipated to be associated with the lower tier of the two-tiered system of resources (an artifact of the 'separate but equal' funding system: the lower tier consists of facilities housing mainly Medicaid residents and, as a result, has very limited resources) (3) greater internal and external barriers to SOPs, (9) no implementation of SOPs, and lower vaccination coverage. (4) Other paths of interest include: internal/external barriers to SOPs; facility staff who were influential in vaccination policy decisions; and a two-tiered system of resources.

The model includes five continuous latent variables. Of the model's three observed non-indicator variables, two were continuous and one (implementation of SOPs) binary.
Also, unstandardized estimates are presented due to model nonlinearity. To properly fit the model, one indicator (i.e., observed variable) was constrained to equal 1 for each latent variable tested. To increase understanding about which indicators loaded highest, we varied the constrained indicator among runs. We do not present a sample covariance matrix due to the presence of a categorical variable. (14) .

Because a binary variable is a dependent variable in the model, we used a robust weighted least squares estimation method which is a weighted least-squares with mean and variance adjustment (WLSMV).(15) Fit criteria included: 1) RMSEA <.06; 2) WRMR which is a weighted average of the residuals - a value of less than 1 ;(16) 3) CFI >.95; 4) Tucker-Lewis Index (TLI)>.95; and 5) $X^{2}$ goodness of fit test, $p>.05$. 


\section{Results}

\subsection{Response Rate}

The response rate for the 2000 survey was $89 \%$ (249 of the 279 eligible nursing homes), and for 2002, the response rate was also $89 \%$ (236 of the 266 facilities). Six of the nursing homes in the second year did not have complete survey information on SOPs or the questions used for the factor analyses, yielding a sample size of 230. However, 234 homes had complete facility data from OSCAR.

\subsection{Descriptive Statistics}

Almost a quarter $(23.5 \%)$ of the nursing homes reported using standing orders for influenza vaccinations in the second year of the study. The most frequently reported 'very significant' barrier to implementing standing orders for immunizations was 'staff lack legal authority to vaccinate without a physician order' (35\% in year 1 and $27 \%$ in year 2) [Table 1]. Among the 234 nursing homes with complete OSCAR data, the median proportion of residents on Medicare was $9.1 \%$ and on Medicaid was $69.9 \%$. Among the 133 (56.8\%) of homes with black residents, themedian proportion of black residents was $4.7 \%$ and the median number of black residents in the facility was 6 .

Table 1. CMS-CDC Standing Orders Project, Facility characteristics by use of SOPs, Vaccination Coverage, and proportion Blacks in the facility, 20012002.

\begin{tabular}{|c|c|c|c|c|c|c|}
\hline & $\begin{array}{l}\text { All } \\
\mathbf{n}=\mathbf{2 3 4}\end{array}$ & $\%$ & $\begin{array}{l}\% \\
\text { blacks } \\
\text { Median } \\
\text { (range) }\end{array}$ & $\begin{array}{l}\text { Influenza coverage } \\
\text { Median } \\
\text { (range) }\end{array}$ & $\begin{array}{l}\text { Sta } \\
\text { ord } \\
\text { vac }\end{array}$ & enza \\
\hline \multicolumn{4}{|l|}{ Ownership } & & & 56 \\
\hline \multicolumn{7}{|l|}{ Profit } \\
\hline Government & 35 & 14 & $0(0-98)$ & $77(38-100)$ & 10 & 18 \\
\hline Non-profit & 74 & 32 & $0(0-98)$ & $69(0-97)$ & 14 & 26 \\
\hline \multicolumn{7}{|l|}{ Certification } \\
\hline Dual certif. & 162 & 70 & $0(0-97)$ & $66(0-100)$ & 37 & 67 \\
\hline Distinct Part & 55 & 23 & $4(0-98)$ & $49(0-84)$ & 13 & 24 \\
\hline SNF- Medicare & 7 & 3 & $4(0-45)$ & $27(0-91)$ & 0 & 0 \\
\hline NF-Medicaid & 10 & 4 & $0(0-12)$ & $82(57-100)$ & 5 & 9 \\
\hline $\begin{array}{l}\text { Chain } \\
\text { Independent }\end{array}$ & 113 & 47 & $0(0-98)$ & $71(0-100)$ & 27 & 49 \\
\hline Multi-facility Chain & 121 & 53 & $1(0-98)$ & $59(0-100)$ & 28 & 51 \\
\hline \multicolumn{7}{|l|}{ Hospital administered } \\
\hline No & 191 & 83 & $1(0-98)$ & $64(0-100)$ & 47 & 85 \\
\hline Yes & 39 & 17 & $0(0-97)$ & $67(0-100)$ & 8 & 15 \\
\hline \multicolumn{7}{|l|}{ Substandard assessments } \\
\hline No & 178 & 76 & $1(0-98)$ & $64(0-100)$ & 44 & 80 \\
\hline Yes & 51 & 22 & $1(0-98)$ & $65(0-100)$ & 11 & 20 \\
\hline \multicolumn{7}{|c|}{ How important are authorities in shaping the facility's immunization policies? } \\
\hline $\begin{array}{l}\text { Director of Nursing } \\
\text { Don't know }\end{array}$ & 1 & $<1$ & 1 & 53 & 0 & 0 \\
\hline Not important & 2 & $<1$ & $9(0-18)$ & $49(6-91)$ & 0 & 0 \\
\hline Somewhat important & 14 & 6 & $0(0-62)$ & $67(0-100)$ & 4 & 7 \\
\hline Important & 78 & 34 & $0(0-97)$ & $62(1-100)$ & 16 & 29 \\
\hline Very important & 13 & 59 & $1(0-98)$ & $66(0-100)$ & 35 & 64 \\
\hline $\begin{array}{l}\text { Infection Control Coordinator } \\
\text { Don't know }\end{array}$ & 5 & 2 & $2(0-62)$ & $43(16-89)$ & 0 & 0 \\
\hline
\end{tabular}




\begin{tabular}{|c|c|c|c|c|c|c|}
\hline & \multicolumn{2}{|l|}{$\begin{array}{l}\text { All } \\
n=234\end{array}$} & \multirow{2}{*}{$\begin{array}{l}\% \\
\text { blacks } \\
\text { Median } \\
\text { (range) }\end{array}$} & \multirow{2}{*}{$\begin{array}{l}\text { Influenza coverage } \\
\text { Median } \\
\text { (range) }\end{array}$} & \multicolumn{2}{|c|}{$\begin{array}{l}\text { Standing } \\
\text { orders for influenza } \\
\text { vaccination } n=55\end{array}$} \\
\hline & $\mathbf{n}$ & $\%$ & & & $\mathbf{n}$ & $\%$ \\
\hline Not important & 4 & 2 & $3(0-7)$ & $57(21-79)$ & 1 & 1 \\
\hline Somewhat important & 13 & 6 & $1(0-87)$ & $66(0-92)$ & 3 & 5 \\
\hline Important & 72 & 31 & $1(0-98)$ & $63(2-100)$ & 13 & 24 \\
\hline Very important & 136 & 59 & $1(0-98)$ & $66(0-100)$ & 38 & 69 \\
\hline \multicolumn{7}{|l|}{ Advisory Committee for Immunization Prac- } \\
\hline tices & 16 & 7 & $1(0-87)$ & $57(17-85)$ & 3 & 5 \\
\hline \multicolumn{7}{|l|}{ Don’t know } \\
\hline Not important & 4 & 2 & $0(0-3)$ & $76(67-92)$ & 0 & 0 \\
\hline Somewhat important & 21 & 9 & $0(0-32)$ & $62(0-100)$ & 6 & 11 \\
\hline Important & 77 & 33 & $1(0-98)$ & $64(0-100)$ & 17 & 31 \\
\hline Very important & 112 & 49 & $1(0-98)$ & $67(0-100)$ & 29 & 53 \\
\hline $\begin{array}{l}\text { Facility Administrator } \\
\text { Don't know }\end{array}$ & 2 & $<1$ & $0(0-1)$ & $66(53-79)$ & 1 & 1 \\
\hline Not important & 16 & 7 & $0(0-18)$ & $69(6-91)$ & 2 & 4 \\
\hline Somewhat important & 27 & 12 & $0(0-15)$ & $66(0-100)$ & 10 & 18 \\
\hline Important & 92 & 40 & $1(0-97)$ & $64(2-100)$ & 19 & 35 \\
\hline Very important & 93 & 40 & $1(0-98)$ & $64(0-92)$ & 23 & 42 \\
\hline $\begin{array}{l}\text { Corporate Leadership } \\
\text { Don't know }\end{array}$ & 35 & 15 & $0(0-33)$ & $73(27-97)$ & 10 & 18 \\
\hline Not important & 26 & 11 & $0(0-86)$ & $73(17-100)$ & 6 & 11 \\
\hline Somewhat important & 31 & 13 & $0(0-32)$ & $62(0-100)$ & 11 & 20 \\
\hline Important & 73 & 32 & $1(0-98)$ & $61(0-100)$ & 15 & 27 \\
\hline Very important & 65 & 28 & $2(0-98)$ & $61(0-89)$ & 13 & 24 \\
\hline $\begin{array}{l}\text { State Health Department } \\
\text { Don't know }\end{array}$ & 13 & 6 & $0(0-81)$ & $61(17-86)$ & 2 & 4 \\
\hline Not important & 13 & 6 & $0(0-5)$ & $64(0-84)$ & 1 & 1 \\
\hline Somewhat important & 31 & 13 & $0(0-45)$ & $58(0-100)$ & 9 & 16 \\
\hline Important & 101 & 44 & $1(0-97)$ & $67(0-100)$ & 23 & 42 \\
\hline Very important & 72 & 31 & $1(0-98)$ & $64(0-97)$ & 20 & 36 \\
\hline $\begin{array}{l}\text { Medical Director } \\
\text { Don't know }\end{array}$ & 1 & $<1$ & 0 & 92 & 1 & 1 \\
\hline Not important & 9 & 4 & $0(0-62)$ & $71(16-91)$ & 2 & 4 \\
\hline Somewhat important & 36 & 16 & $1(0-62)$ & $60(0-100)$ & 4 & 7 \\
\hline Important & 93 & 40 & $1(0-98)$ & $64(0-100)$ & 24 & 44 \\
\hline Very important & 91 & 40 & $1(0-97)$ & $65(0-100)$ & 24 & 44 \\
\hline $\begin{array}{l}\text { Quality Improvement Organization } \\
\text { Don't know }\end{array}$ & 31 & 13 & $1(0-31)$ & $62(0-96)$ & 4 & 7 \\
\hline Not important & 11 & 5 & $0(0-32)$ & $78(21-91)$ & 1 & 1 \\
\hline Somewhat important & 36 & 16 & $0(0-87)$ & $65(0-100)$ & 11 & 20 \\
\hline Important & 107 & 47 & $1(0-98)$ & $64(0-100)$ & 24 & 44 \\
\hline Very important & 45 & 20 & $1(0-97)$ & $64(0-92)$ & 15 & 27 \\
\hline $\begin{array}{l}\text { State Certification Surveyor } \\
\text { Don't know }\end{array}$ & 14 & 6 & $0(0-7)$ & $61(0-96)$ & 2 & 4 \\
\hline Not important & 20 & 9 & $0(0-62)$ & $72(0-91)$ & 4 & 7 \\
\hline Somewhat important & 30 & 13 & $0(0-81)$ & $56(0-92)$ & 13 & 24 \\
\hline Important & 83 & 36 & $1(0-98)$ & $63(0-100)$ & 13 & 24 \\
\hline Very important & 83 & 36 & $1(0-98)$ & $66(4-97)$ & 23 & 42 \\
\hline \multicolumn{7}{|c|}{ How significant is this barrier to prevent implementation of standing order programs for vaccinating residents of your facility? } \\
\hline $\begin{array}{l}\text { Doctors need education about standing orders } \\
\text { Don't know }\end{array}$ & 9 & 4 & $0(0-9)$ & $66(21-96)$ & 2 & 4 \\
\hline Not significant & 117 & 51 & $0(0-98)$ & $68(0-100)$ & 39 & 71 \\
\hline Somewhat significant & 35 & 15 & $2(0-92)$ & $65(0-100)$ & 4 & 7 \\
\hline Significant & 45 & 20 & $1(0-97)$ & $57(0-100)$ & 6 & 11 \\
\hline Very significant & 24 & 10 & $3(0-97)$ & $62(0-88)$ & 4 & 7 \\
\hline \multicolumn{7}{|l|}{ Concerns about medical liability for the facili- } \\
\hline ty & 16 & 7 & $0(0-62)$ & $67(16-96)$ & 2 & 4 \\
\hline \multicolumn{7}{|l|}{ Don’t know } \\
\hline Not significant & 106 & 46 & $0(0-98)$ & $66(0-100)$ & 33 & 60 \\
\hline Somewhat significant & 56 & 24 & $1(0-97)$ & $63(0-100)$ & 13 & 24 \\
\hline Significant & 30 & 13 & $0(0-97)$ & $57(1-100)$ & 6 & 11 \\
\hline Very significant & 22 & 10 & $3(0-98)$ & $64(34-86)$ & 1 & 1 \\
\hline
\end{tabular}




\begin{tabular}{|c|c|c|c|c|c|c|}
\hline & \multicolumn{2}{|l|}{$\begin{array}{l}\text { All } \\
\mathrm{n}=\mathbf{2 3 4}\end{array}$} & \multirow{2}{*}{$\begin{array}{l}\% \\
\text { blacks } \\
\text { Median } \\
\text { (range) }\end{array}$} & \multirow{2}{*}{$\begin{array}{l}\text { Influenza coverage } \\
\text { Median } \\
\text { (range) }\end{array}$} & \multicolumn{2}{|c|}{$\begin{array}{l}\text { Standing } \\
\text { orders for influenza } \\
\text { vaccination } n=55\end{array}$} \\
\hline & $\mathbf{n}$ & $\%$ & & & $\mathbf{n}$ & $\%$ \\
\hline $\begin{array}{l}\text { Cost of program } \\
\text { Don't know }\end{array}$ & 12 & 5 & $1(0-19)$ & $64(21-96)$ & 1 & 1 \\
\hline Not significant & 146 & 63 & $1(0-98)$ & $67(0-100)$ & 40 & 73 \\
\hline Somewhat significant & 30 & 13 & $1(0-86)$ & $59(0-91)$ & 9 & 16 \\
\hline $\begin{array}{l}\text { Significant } \\
\text { Very significant }\end{array}$ & $\begin{array}{l}29 \\
13\end{array}$ & $\begin{array}{l}13 \\
6\end{array}$ & $\begin{array}{l}0(0-97) \\
1(0-98)\end{array}$ & $\begin{array}{l}57(2-91) \\
46(0-86)\end{array}$ & $\begin{array}{l}3 \\
2\end{array}$ & $\begin{array}{l}5 \\
4\end{array}$ \\
\hline \multicolumn{4}{|c|}{ Concerns about inappropriately vaccinating } & $72(0-96)$ & 2 & 4 \\
\hline $\begin{array}{l}\text { Don't know } \\
\text { Not significant }\end{array}$ & 91 & 40 & $0(0-97)$ & $67(0-96)$ & 28 & 51 \\
\hline Somewhat significant & 63 & 27 & $1(0-97)$ & $60(4-100)$ & 16 & 29 \\
\hline Significant & 37 & 16 & $1(0-97)$ & $63(0-100)$ & 6 & 11 \\
\hline Very significant & 29 & 13 & $4(0-98)$ & $61(0-89)$ & 3 & 5 \\
\hline $\begin{array}{l}\text { Lack of support of facil } \\
\text { Don't know }\end{array}$ & 10 & 4 & $0(0-9)$ & $67(21-96)$ & 2 & 4 \\
\hline Not significant & 155 & 67 & $1(0-98)$ & $66(0-100)$ & 43 & 78 \\
\hline Somewhat significant & 26 & 11 & $0(0-18)$ & $59(6-100)$ & 5 & 9 \\
\hline Significant & 25 & 11 & $1(0-97)$ & $63(0-100)$ & 4 & 7 \\
\hline Very significant & 14 & 6 & $4(0-98)$ & $64(0-91)$ & 1 & 1 \\
\hline \multicolumn{7}{|l|}{ Low reimbursement } \\
\hline Don’t know & 23 & 10 & $2(0-62)$ & $62(0-96)$ & 4 & 7 \\
\hline Not significant & 145 & 63 & $1(0-98)$ & $67(0-100)$ & 43 & 78 \\
\hline Somewhat significant & 28 & 12 & $0(0-86)$ & $58(7-91)$ & 3 & 5 \\
\hline Significant & 22 & 10 & $0(0-97)$ & $58(2-86)$ & 3 & 5 \\
\hline Very significant & 12 & 5 & $3(0-98)$ & $53(0-83)$ & 2 & 4 \\
\hline \multicolumn{7}{|l|}{ Other priorities of staff } \\
\hline Don’t know & 11 & 5 & $1(0-9)$ & $69(16-96)$ & 2 & 4 \\
\hline Not significant & 111 & 48 & $1(0-98)$ & $66(0-100)$ & 32 & 58 \\
\hline Somewhat significant & 43 & 19 & $0(0-86)$ & $66(14-92)$ & 11 & 20 \\
\hline Significant & 42 & 18 & $0(0-98)$ & $61(0-100)$ & 6 & 11 \\
\hline Very significant & 23 & 10 & $0(0-92)$ & $64(0-91)$ & 4 & 7 \\
\hline \multicolumn{7}{|c|}{ Staff lack legal authority to vaccinate without } \\
\hline \multicolumn{7}{|l|}{ Don't know } \\
\hline Not significant & 77 & 33 & $0(0-98)$ & $68(7-97)$ & 32 & 58 \\
\hline Somewhat significant & 25 & 11 & $1(0-32)$ & $64(0-100)$ & 7 & 13 \\
\hline Significant & 51 & 22 & $1(0-98)$ & $60(0-100)$ & 4 & 7 \\
\hline Very significant & 63 & 27 & $2(0-92)$ & $62(0-100)$ & 9 & 16 \\
\hline $\begin{array}{l}\text { Facility has written pr } \\
\text { policy }\end{array}$ & 56 & 23 & $0(0-86)$ & $64(0-97)$ & 11 & 20 \\
\hline \multicolumn{7}{|l|}{ No } \\
\hline Yes & 178 & 77 & $1(0-98)$ & $64(0-100)$ & 44 & 80 \\
\hline
\end{tabular}

\subsection{Structural Equation Models}

All the fit statistics indicated the structural equation model was a good fit. The RMSEA was 0.023 , the WRMR was 0.700 , the CFI was 0.967 , the TLI was 0.957 , and the
$\mathrm{X} 2$ goodness of fit test was 219.43 , $\mathrm{df}=196, \mathrm{p}=0.1205$. The final model included five latent variables identified in the factor analyses; all the indicators of the latent variables had statistically significant loadings. 
For the latent variable related to influential facility staff in vaccination policy decisions, the medical director was most important (1.61, SE: .28), followed by the facility administrator (1.45, SE: .19). For the latent variable related to influential outside authoritative bodies the Quality Improvement Organization was most important (1.76, (SE: .37), followed by the state certificationsurveyor (1.51, SE: .32).

Two latent variables were confirmed that indicated barriers to implementing standing orders. For the latent variable related to internal facility barriers to SOPs, lack of support of facility leadership was most important (1.18, SE: .20), followed by low reimbursement (1.08, SE: .19). For the latent variable related toxternal facility (i.e., legal and liability) barriers to SOPs, doctors need education about SOPs was most important (1.51, SE: .25), followed by staff lack the legal authority to immunize without resident's physician order (1.45, SE: .28).

Estimates of the final measurement portion of the model are in Figure 3 and estimates of the structural portion of the model are in Figure 4. Statistically significant paths have bolded arrows and italicized estimates. In the structural model, vaccination coverage was 7.7 percentage points higher in facilities with SOPs compared with facilities without SOPs $(p=.001)$. The latent variable, external facility barriers, associated as a significant barrier to implementing SOPs $(\mathrm{p}=.001)$. For every one unit increase in the latent variable of external facility barriers (the higher the estimate for the latent variable the higher the probability of concern), the odds of implementing SOPs decreased by 0.51 . Higher proportions of black residents significantly associated with the latent variable, internal facility barriers to SOPs $(p=.018)$. The latent variable, external facility concerns as perceived barriers to SOPs, significantly associated with both latent variables for influential authorities in vaccination policy decisions: with authoritative body $(p=.012)$ and facility staff $(\mathrm{p}<.001)$. The latent variable, influential senior staff who make vaccination policy decisions, significantly associated with the latent variable, influential outside authoritative bodies who make vaccination decisions $(\mathrm{p}<.001)$.

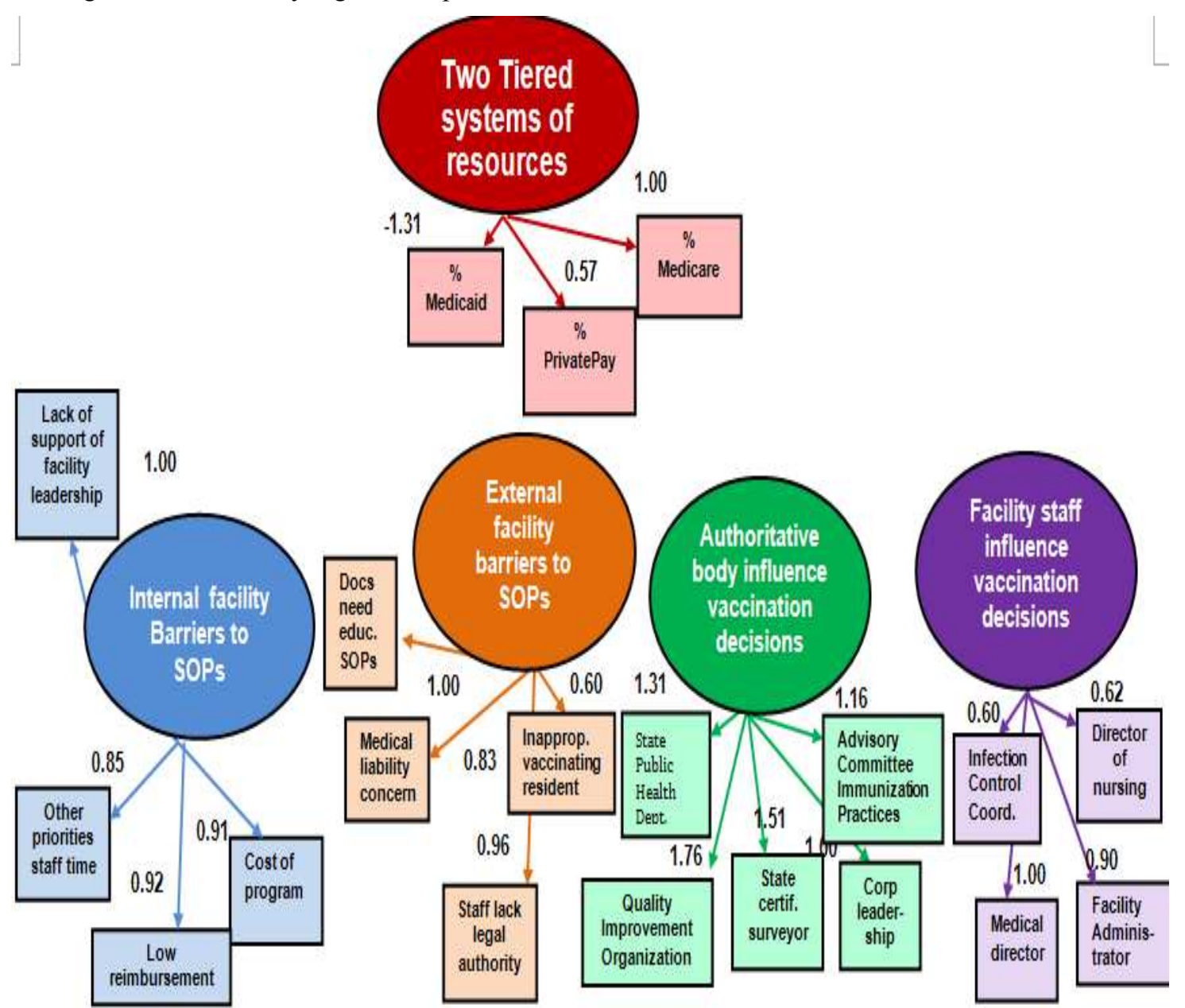

Figure 3. Measurement model: Barriers to implementing standing orders for vaccinations, influentialauthorities for vaccination policies and two-tiered system of resources. 


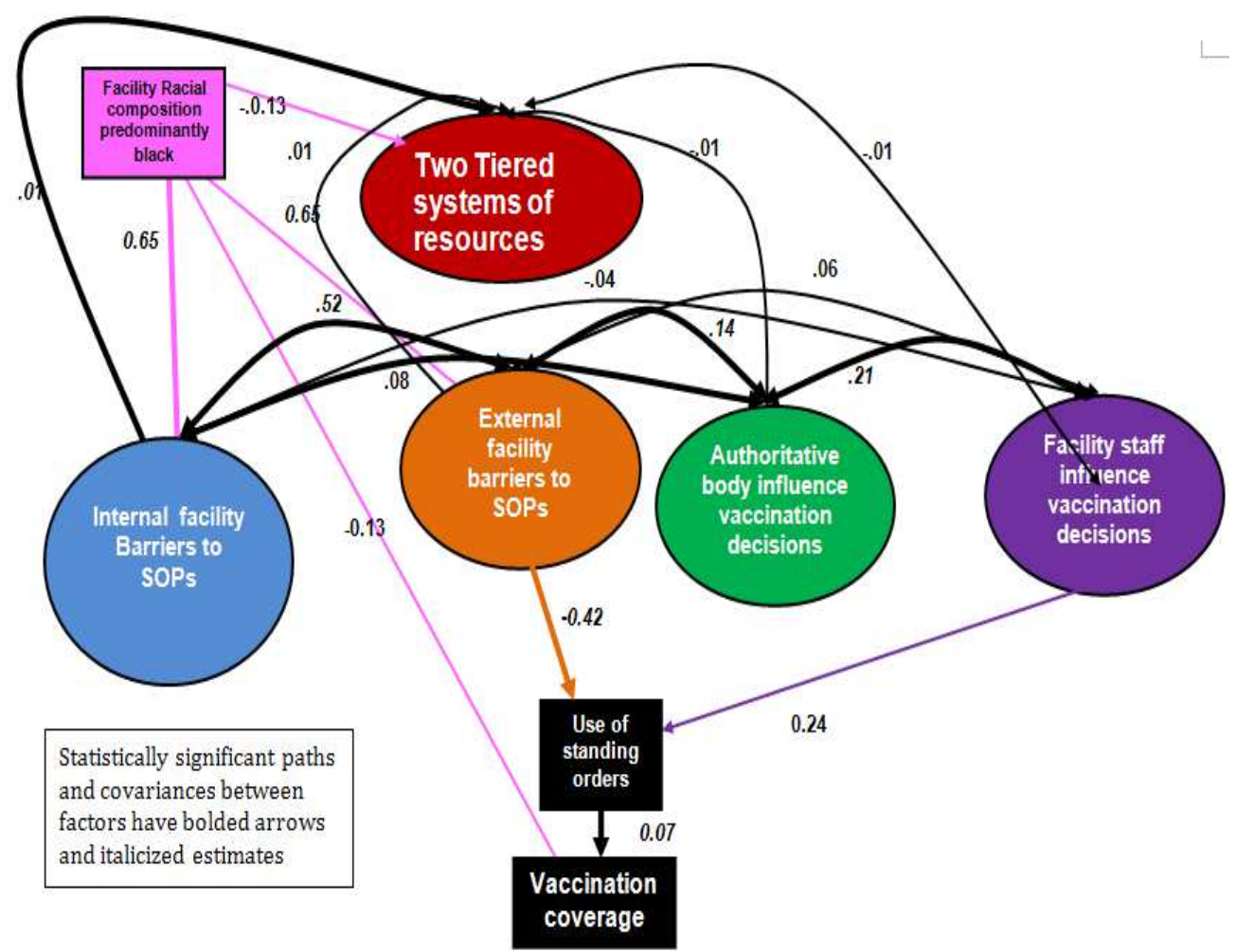

Figure 4. Structural Model: Racial composition of the nursing home, barriers to implementing standing orders for vaccinations, and influential authorities for vaccination policies jointly aciated with use of SOPs and nursing home-level vaccination coverage.

\section{Discussion}

As hypothesized, external facility concerns, which included concern for liability and legal issues, negatively associated with implementing SOPs; but this association may be mitigated to some degree by the influence of outside bodies on vaccination policies and senior staff.

The outside authoritative body that had the most influence on shaping vaccination policy was the QIO, followed by the state certification surveyor. Therefore those authorities are best positioned to address the perceived barriers especially the fear that a physician signature is needed before a service is delivered or that they will be at risk for litigation. Indeed, there may have been a perception on the part of nursing home staff at the time this project was conducted that SOPs constituted 'bad patient care', at least partly because of the presence of a federal law that Medicare would not reimburse the cost of vaccination in facilities that used SOPs for vaccination.Another program, the National Vaccine Injury Compensation Program, which is a no-fault dispute resolution system for resolving vaccine injury claims that was established in 1988 to compensate individuals and families injured by vaccines, was also present at the time of this project.(18)Educating staff on this program could at least partly assuage some of these concerns about medical liability for the nursing home. Even in facilities where a physician signature is eventually needed for all orders, this does not preclude implementing an SOP, e.g., in this case the order would be signed after delivery of the service or vaccine. Messages to address theseissues should be clearly delivered by the QIO and state certification surveyor.

The external concern about doctors in need of education about SOPs may have to do with physician unwillingness to give up the need for their signature for vaccination. For example, physicians' may have concerns that SOPsapply too broad an approach, inappropriately targeting some individuals for vaccine who do not need it or who have avaccine contraindication. Doctors in need of education about SOPs and staff lacking legal authority were the two greatest contributors to the underlying latent variable, external barriers to implementing SOPs. At the time of this study 43 states and the District of Columbia permitted the use of standing orders as recommended by the ACIP.(19) Thus these concerns deserve further research to understand their relative impact and how to remove them as barriers to improving SOP utilization.

Internal facility barriers such as perceived low reimbursement and the cost of implementing the program to the facility did not associate with the implementation of SOPs in our model. Instead, internal barriers led to a more poorly fitting model and therefore were not included as a path to implementation of SOPs. As mentioned above, at the time of our study, CMS (Medicare) did not reimburse nursing homes with SOPs for the influenza vaccine. Importantly, CMS removed that policy in October, 2002 and has since reimbursed facilities that have SOPs for vaccinations.(20).

The proportion of black residents in the facility was associated with bothinternal and external barriers to imple- 
menting SOPs. Other studies have found the higher the proportion of black residents in the nursing home the lower the likelihood of being offered the vaccine as well as receiving the vaccine.(4) Thus this finding highlights the importance of addressing barriers to implementing SOPs in nursing homes, particularly in the predominantly black homes. Also, the higher the proportion of black residents in the nursing home the higher the likelihood of perceived barriers related to the cost of the program, low reimbursement, and other priorities of staff time. This finding suggests that the predominantly black homes are likely to be poorly resourced nursing homes. In another analysis we found that the nursing homes with higher proportions of black residents had lower ratios of FTEs to beds and lower $\mathrm{RN}$ and direct-care staff hours per resident day. (CDC, unpublished).

Vaccination coverage was higher among facilities with SOPs (7.7 percentage points) than facilities without SOPs. In our national study,(8)the adjusted difference in vaccination coverage for residents in homes with standing orders compared to those without standing orders was modest-only 4.7 compared with the 7.7 percentage points that we report here. Although higher vaccination coverage was reported among nursing homes with SOPs, it was below what we had hypothesized,which was an increase of at least $10 \%$. In a previous analysis of this study, we found facilities that adopted SOPs after the QIO interventions did not significantly increase their vaccination coverage by $10 \%$ or more from one year to the next.(11)This may indicate that SOPs are often poorly implemented, or that the QIO intervention itself was effective in increasing vaccination rate independent of whether it led to the implementation of an SOP.

\section{Limitations}

A limitation of the study is the small ratio of "sample size to number of parameters" in the model. We may have failed to detect statistically significant paths in our best fitting structural model due to lack of power. However, because the statistically non-significant paths are theoretically sound and the model was a good fit, it is reasonable to assume those paths are important in the model overall; future research should investigate these paths. Another limitation of our data is the number of responses of 'don't know' to the questions on influential vaccination authorities and barriers to implementing SOPs (highest proportion is $15 \%$ of the responses). We assumed that if the respondent did not know then that factor was very unimportant or highly insignificant. However, it could be that the factor was indeed important or significant although the respondent was not aware. Also, although some of the variables in our analyses were individual level variables that were aggregated to the facility level (e.g., proportion black residents and vaccination coverage), the research questions were intended to make inferences at the facility level. We were unable to adjust for resident-level characteristics but we would not expect such factors to affect facility-level authorities who shape decisions for vaccination or barriers to implementation of SOPs. Finally, although we tried to define SOPs clearly, the definition of SOP varies widely among healthcare providers and therefore may have led to some misclassification.

\section{Conclusions}

This study highlights the importance of perceptions of concerns such as staff lacking legal authority for standing orders. Our findings also confirm that nursing homes with SOPs are associated with higher vaccination coverage than homes without such programs after controlling for barriers. Further, authorities such as QIOs and the state certification surveyor were found to be significantly influential in shaping vaccination policies in nursing homes. Although QIOs' work with nursing homes vary,these findings strengthen the case for QIOs and the state certification surveyor to address perceived concerns by staff who shape vaccination policies in the nursing homes, such as the Medical Director and Facility Administrator, as found in our results.

\section{Acknowledgements}

We would like to thank Mary Mason McCauley, MTSC, for help with editing. The authors have no conflicts of interest. We certify that, there are no conflicts of interest for any of the authors.

\section{Required Disclaimer}

The analyses upon which this publication is based were performed under Intra-agency Agreement Number IA07-52 between the Centers for Disease Control and Prevention (CDC) and the Centers for Medicare \& Medicaid Services (CMS), Department of Health and Human Services. The content of this publication does not necessarily reflect the views or policies of the Department of Health and Human Services, nor does mention of trade names, commercial products, or organizations imply endorsement by the U.S. Government. The authors assume full responsibility for the accuracy and completeness of the ideas presented. Ideas and contributions to the authors concerning experience in engaging with issues presented are welcomed.

\section{References}

[1] Bardenheier B, Wortley PM, Ahmed F, Shefer A, Hales C. Influenza immunization coverage among residents of longterm care facilities certified by CMS, 2005-2006: The newest MDS quality indicator. Journal of the American Medical Directors Association 2010;11:59-69.

[2] Bardenheier B, Wortley PM, Shefer A. Influenza vaccine among African-American and white nursing home residents: Is there a gap? J Am Geriatr Soc 2009;57:2164-5.

[3] Mor V, Zinn J, Angelelli J, Teno JM, Miller SC. Driven to 
tiers: socioeconomic and racial disparities in the quality of nursing home care. Milbank Q 2004;82:227-56.

[4] Bardenheier B, Wortley PM, Ahmed F, Gravenstein S, Hogue CR. Racial inequities in receipt of influenza vaccination among long-term care residents within and between facilities in Michigan. Med Care 2011;49:371-7.

[5] Use of Standing Orders Programs to Increase Adult Vaccination Rates. MMWR Morb Mortal Wkly Rep 2000 Mar 24;49(RR-1):15-26.

[6] Recommendations regarding interventions to improve vaccination coverage in children, adolescents, and adults. Task Force on Community Preventive Services. Am J Prev Med 2000 Jan;18(1 Suppl):92-6.

[7] The Community Preventative Services Task Force. Universally Recommended Vaccinations: CommunityBased Interventions Implemented in Combination. The Community Preventive Services Task Force 2012 April 10.

[8] Bardenheier B, Shefer A, Lu P, Remsburg R, Marsteller J. Are standing order programs associated with influenza vaccination? - NNHS, 2004. J Am Med Dir Assoc 2010;11:654-61.

[9] Shefer A, McKibben L, Bardenheier B, Bratzler D, Roberts $\mathrm{H}$. Characteristics of long-term care facilities associated with standing order programs to deliver influenza and pneumococcal vaccinations to residents in 13 states. J Am Med Dir Assoc 2005;6:97-104.

[10] Bardenheier B, Shefer A, Ahmed A, Remsburg R, Hogue $\mathrm{CR}$, Gravenstein S. Do racial inequities in receipt of influenza vaccination among nursing home residents narrow if facility vaccination policies exist? Results of the National Nursing Home Survey, 2004. J Am Geriatr Soc 2011;59:687-93.

[11] Bardenheier BH, Shefer A, McKibben L, Roberts H, Rhew D, Bratzler D. Factors Predictive of Increased Influenza and Pneumococcal Vaccination Coverage in Long-term Care Facilities: The CMS-CDC Standing Orders Program Project.
J Am Med Dir Assoc 2005;6:291-9.

[12] Centers for Medicare \& Medicaid Services. State Operations Manual. Baltimore, MD: Appendix Q 42. CFR 483.25 Quality of Care; 1999.

[13] Institute of Medicine. Improving the quality of long-term care. Washington D.C.: National Academy Press; 2001.

[14] Principles and practice of structural equation modeling. 3rd ed. New York, NY: The Guilford Press; 2010.

[15] Huba GJ, Harlow LL. Robust Structural Equation Models: Implications for developmental psychology. Child Development 1987;58:147-66.

[16] Muthén BO, du Toit S, Spisic D. Robust inference using weighted least squares and quadratic estimating equations in latent variable modeling with categorical and continuous outcomes. University of California LA, editor. 1997.

[17] Yu C, Muthén BO. Evaluation of model fit indices for latent variable models with categorical and continuous outcomes. New Orleans, LA: American Educational Research Association; 2002.

[18] U.S.Department of Health and Human Services. National Vaccine Injury Compensation Program (VICP). Health Resources and Services Administration 2011 [cited 2011 Feb. 4] ; Available from: URL: http://www.hrsa.gov/vaccinecompensation/index.html.

[19] Devore E NSDL. State Policies, Laws and Regulations Governing Immunizations in Long-Term Care Facilities. National Conference of State Legislatures; 2001.

[20] Centers for Medicare \& Medicaid Services. Medicare and Medicaid Programs; Conditions of Participation:Immunization Standards for Hospitals,LongTerm Care Facilities, and Home Health Agencies. Federal Register 2002;67:61808-14.

[21] Loehlin JC. Latent Variable Models: An introduction to factor, path, and structural equation analysis. 4th ed. Mahwah, NJ: Lawrence Erlbaum Associates; 2004. 